\title{
Application of the Recent ARF1 Calibration Measurements to the Pbar RF Curve Loading Console Application (P2)
}

\author{
Steve Werkema
}

\begin{abstract}
This report documents the determination of the $\mathrm{P} 2 \mathrm{ARF} 1$ constants from the calibration measurements that were made on April 18, 2001? In addition, this report will compare the measured response of ARF1 to that derived from the present P2 constants. This comparison will be used to explain some resent observations of ARF1 behavior.
\end{abstract}

\section{Introduction}

The P2 console application (Antiproton Source RF Files) calculates frequency and voltage curves from a sequence of command statements input by the user. P2 initially calculates these curves in terms of the actual frequencies and voltages required on the RF cavity as a function of time. These curves are then converted to the appropriate low-level drive voltages that will cause the RF system high-level electronics to generate the required frequency and voltage ramps. The low-level drive ramps are then downloaded into CAMAC ramp cards.

In order to convert the required cavity voltage and frequency into the correct drive voltages P2 uses a set of constants that determined from calibration measurements of the various Antiproton Source RF systems. These constants are editable from the P2 constants window. The P2 constants at the time of this writing are shown in Figure 1. The validity of these constants determines the extent to which P2 able to translate the user's commands into the actual voltages and frequencies that appear on the RF cavity. A comparison of the 4/18/2001 calibration of ARF 1 with that presently assumed by $\mathrm{P} 2$ shows a large discrepancy in both the frequency constants and the voltage constants.

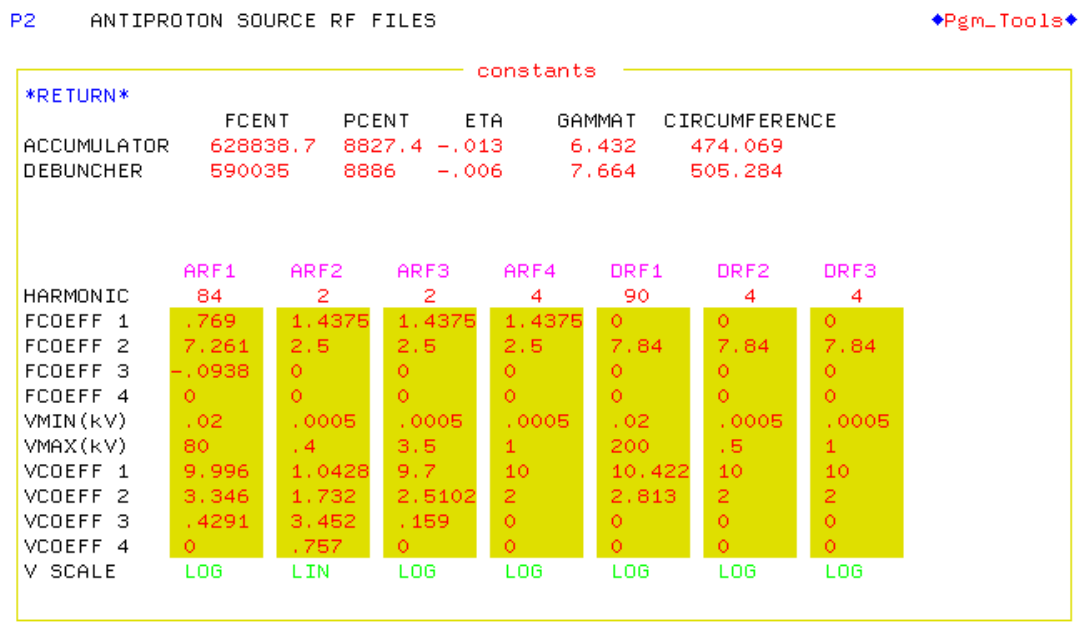

Figure 1 P2 Constants window showing the calibration constants presently in use. The FCOEFF constants are the coefficients of a $3^{\text {rd }}$ order polynomial used to calculate the frequency drive. The VCOEFF numbers are the coefficients of a $3^{\text {rd }}$ order polynomial used to calculate the voltage drive.

\footnotetext{
${ }^{1} \mathrm{P} 2$ is the Antiproton Source RF curve calculation and loading console application.

${ }^{2}$ See Pbar Note 659 by Ralph Pasquinelli
} 


\section{Voltage Calibration}

In its calculation of the voltage ramp, $\mathrm{P} 2$ determines the low-level drive voltage, $V_{d}$, from the requested cavity voltage, $V_{c}$, by the following formula:

$$
V_{d}\left(V_{c}\right)=V C O E F F 1+V C O E F F 2 \cdot z+V C O E F F 3 \cdot z^{2}+V C O E F F 4 \cdot z^{3}
$$

where, $z=\log _{10}\left(\frac{V_{c}}{V_{\max }}\right)$.

VCOEFF1 through VCOEFF4 and $V_{\max }$ used in equation (1) are the values shown in the constants window (see Figure 1).

The calibration of ARF1 provides measurements of cavity voltage (measured from the AP50 fanbacks) versus low-level drive voltage. Fitting this data to a cubic polynomial yields the required $V C O E F F$ values. Figure 2 shows a plot of drive voltage $\left(V_{d}\right)$ versus z.

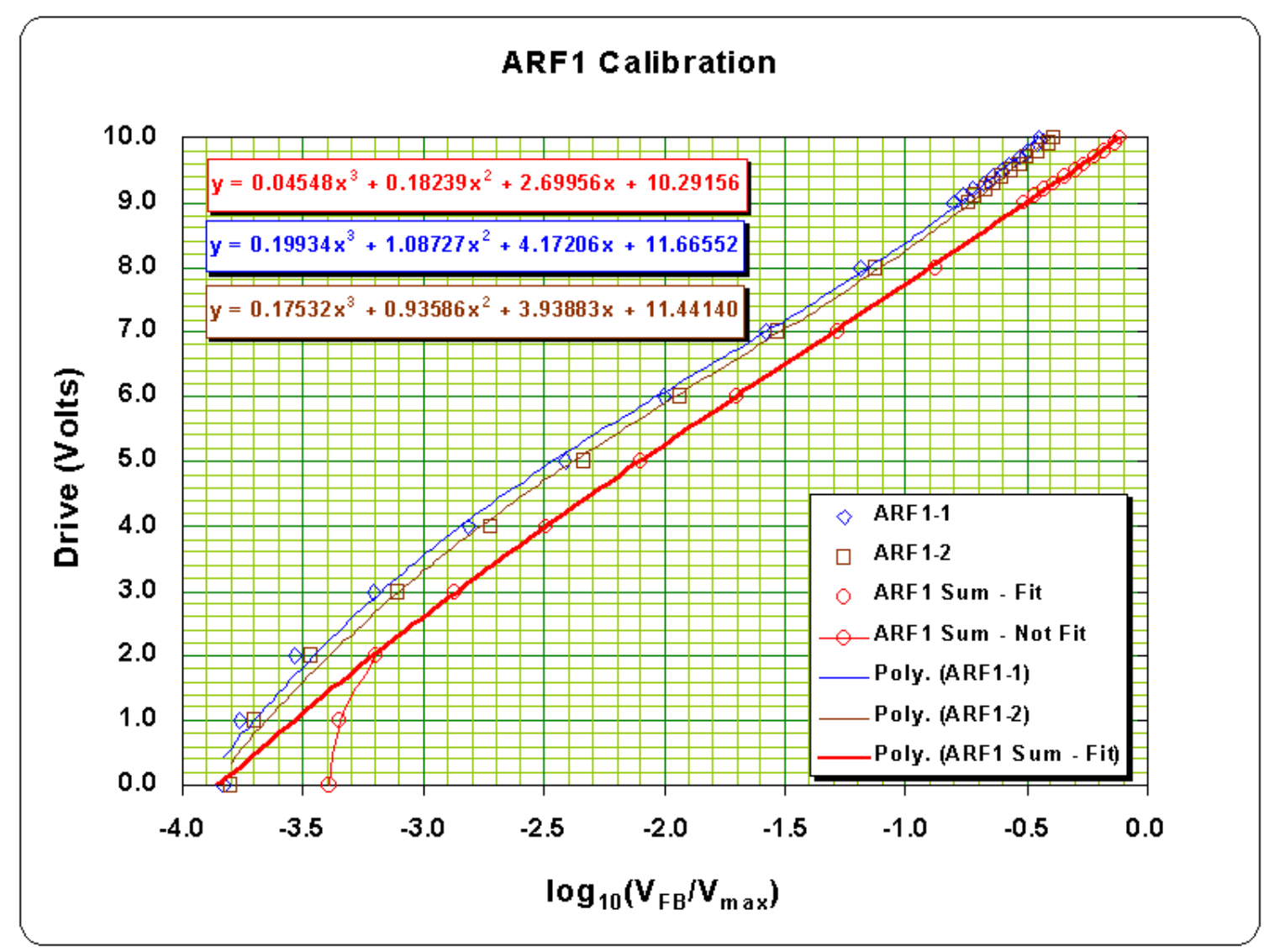

Figure $2 V_{d}$ versus $z$ from the April 18, 2001 ARF1 calibration measurements. $V_{F B}$ is the cavity voltage fanback measured in the AP50 service building. The fanbacks of the individual cavities (ARF1-1 and ARF1-2) were measured as well as the fanback sum.

The ARF1 high-level electronics drives two cavities (ARF1-1 and ARF1-2). Since the lowlevel drives both cavities from a single 164 ramp card, the fit to the fanback sum measurement is used to determine the $\mathrm{P} 2$ VCOEFF values. The required $V C O E F F$ values are simply the coefficients shown in the fit to the fanback sum in Figure 2. These coefficients are compared with the present P2 calibration in Table 1. 
The cubic fit accurately represents the high voltage response. The measurements corresponding to $V_{d}$ less than 2 Volts were not included in the fit.

Table 1 Old and New P2 ARF1 Voltage Coefficients

\begin{tabular}{|c|c|c|}
\hline & Old P2 Constants & New P2 Constants \\
\hline VCOEFF1 & 9.9960 & 10.29156 \\
VCOEFF2 & 3.3460 & 2.69956 \\
VCOEFF3 & 0.4291 & 0.18239 \\
VCOEFF4 & 0.0000 & 0.04548 \\
\hline
\end{tabular}

The effect of implementing the new P2 voltage calibration constants is illustrated in Figure 3 . This figure graphs $V_{c}$ versus $V_{d}$ by inverting equation (1) for both sets of voltage coefficients in Table 1. It is clear that the present (i.e. old) P2 calibration constants differ significantly from the actual response of ARF1 to its low-level drive.

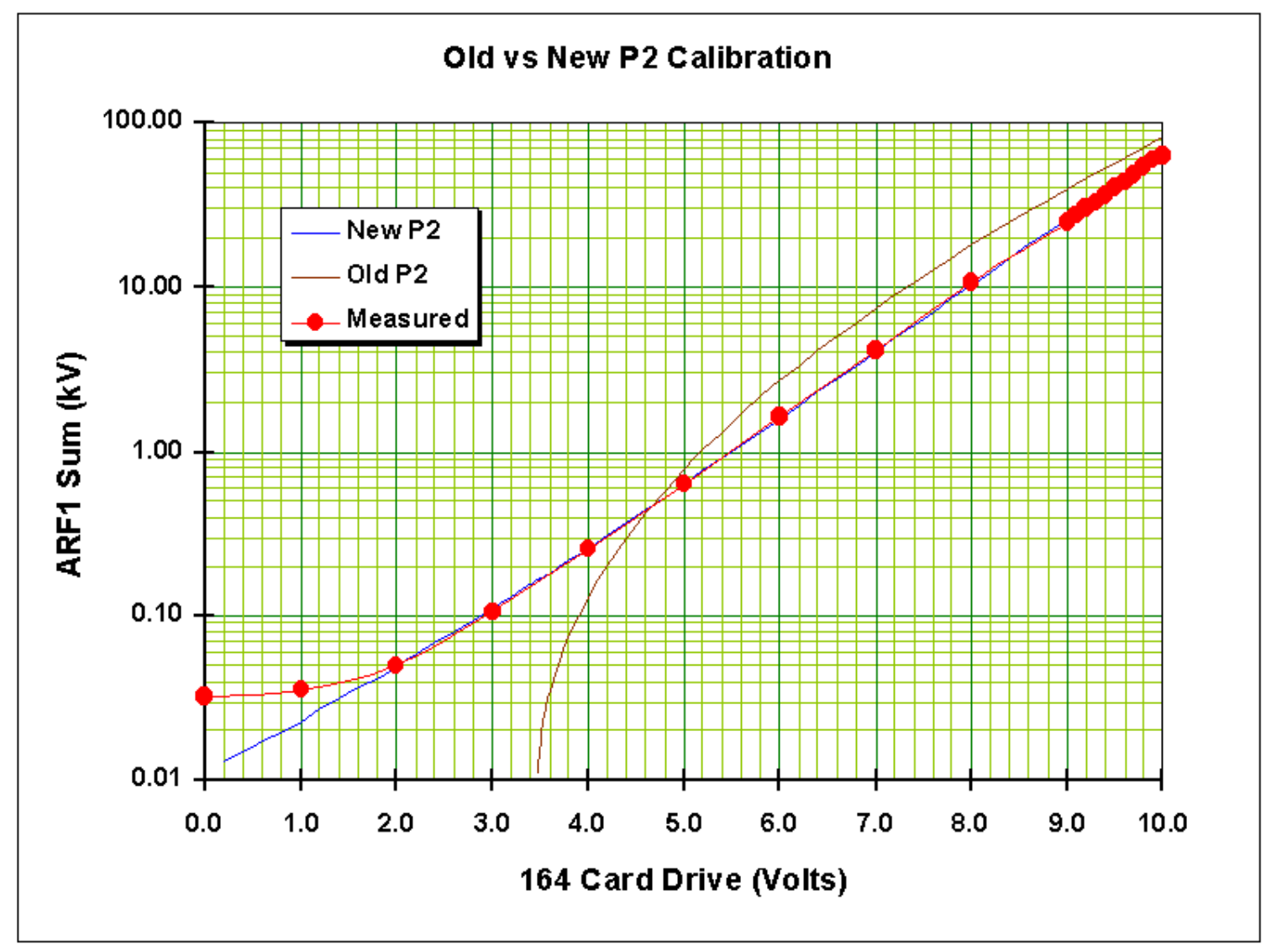

Figure 3 Comparison of the old P2 calibration (i.e. the constants from Figure 1 and the new constants. The Old and New P2 curves are obtained by solving equation (1) for $V_{c}$. The $V_{c}$ versus $V_{d}$ measurements of the April 18,2001 calibration is also plotted.

Perhaps the most egregious discrepancy in the present P2 calibration is its behavior for $V_{d}<4.5$ Volts. During an up-ramp, $\mathrm{P} 2$ will calculate a curve that begins with an initial drive voltage that corresponds to a cavity voltage given by $V_{\min }(20$ Volts - see Figure 1$)$. The 
graph of the old P2 calibration shows that this $V_{\min }$ corresponds to a drive of about 3.5 Volts. The actual calibration shows that this amount of drive results in an actual voltage of about 170 Volts applied to the cavities. Similarly, on a down-ramp, the ramp is ended when a cavity voltage of $V_{\min }$ is reached - the old P2 calibration will leave about 170 Volts on the cavities until the system is gated off. This behavior explains the apparent "pedestal" that has been observed in all P2 calculated ARF1 voltage curves. From this difficulty, the following rule governing the determination of $\mathrm{P} 2$ voltage constants is prescribed:

\section{Thou shalt never enter $P 2$ voltage constants that fail to give reasonable values of $V_{c}$ for all drive voltages between 0 and 10 Volts ${ }^{4}$.}

\section{Frequency Calibration}

P2 calculates the low-level drive voltage, $V_{d}$, for frequency ramps from the requested revolution harmonic of the cavity drive, $f_{\text {rev }}$, by the following formula:

$$
V_{d}\left(f_{r e v}\right)=F C O E F F 1+F C O E F F 2 \cdot z+F C O E F F 3 \bullet z^{2}+F C O E F F 4 \bullet z^{3}
$$

where, $z=\frac{f_{\text {rev }}-f_{\text {cent }}}{\Delta f_{\max }}$.

FCOEFF1 through FCOEFF4 and $f_{\text {cent }}$ used in equation (2) are the values shown in the constants window (see Figure 1). $\Delta f_{\max }$ is a number that is hard-coded into $\mathrm{P} 2$ and has a value given by: $\Delta f_{\max }=200 \mathrm{~Hz}^{2}$.

As with the voltage calibration, the $F C O E F F$ values can be determined by fitting the measurements of RF frequency versus drive voltage to a polynomial. This is done in Figure 4. The response of RF frequency to the low-level drive is linear over the range of frequencies used by ARF1; therefore, the calibration data is fit to a straight line. The present FCOEFF values and those derived from Figure 4 are given in Table 2.

Table 2 Old and New P2 ARF1 Frequency Coefficients

\begin{tabular}{|c|r|r|}
\hline & Old P2 Constants & New P2 Constants \\
\hline FCOEFF1 & 0.7690 & 0.44517 \\
FCOEFF2 & 7.2610 & 7.44384 \\
FCOEFF3 & -0.0938 & 0.00000 \\
FCOEFF4 & 0.0000 & 0.00000 \\
\hline
\end{tabular}

A comparison of the old and new P2 frequency calibration is given in Figure 5. This graph shows that P2 presently under-estimates the drive required to generate a given frequency on the cavity. Moreover, the magnitude of the error grows as $f_{\text {rev }}$ is changed from the Accumulator injection orbit $(628765 \mathrm{~Hz})$ toward the central orbit $(628840 \mathrm{~Hz})$. The variation of the frequency error is shown in Figure 6. This P2 calibration error explains the lack of correspondence between the frequencies requested and those measured on the ARF1 fanbacks during pbar stacking.

\footnotetext{
${ }^{3}$ Reasonable values are cavity voltages that are positive and bear at least some resemblance to an actual calibration of the RF system.

${ }^{4}$ Even though the ramp cards in use in the Antiproton Source are capable of outputs that range from -10 Volts to

+10 Volts, the high level electronics generally only accepts positive drive voltages.

${ }^{5} \Delta f_{\max }$ is chosen so that $|z|<1$ for all frequencies that will be used in Accumulator and Debuncher RF ramps.
} 


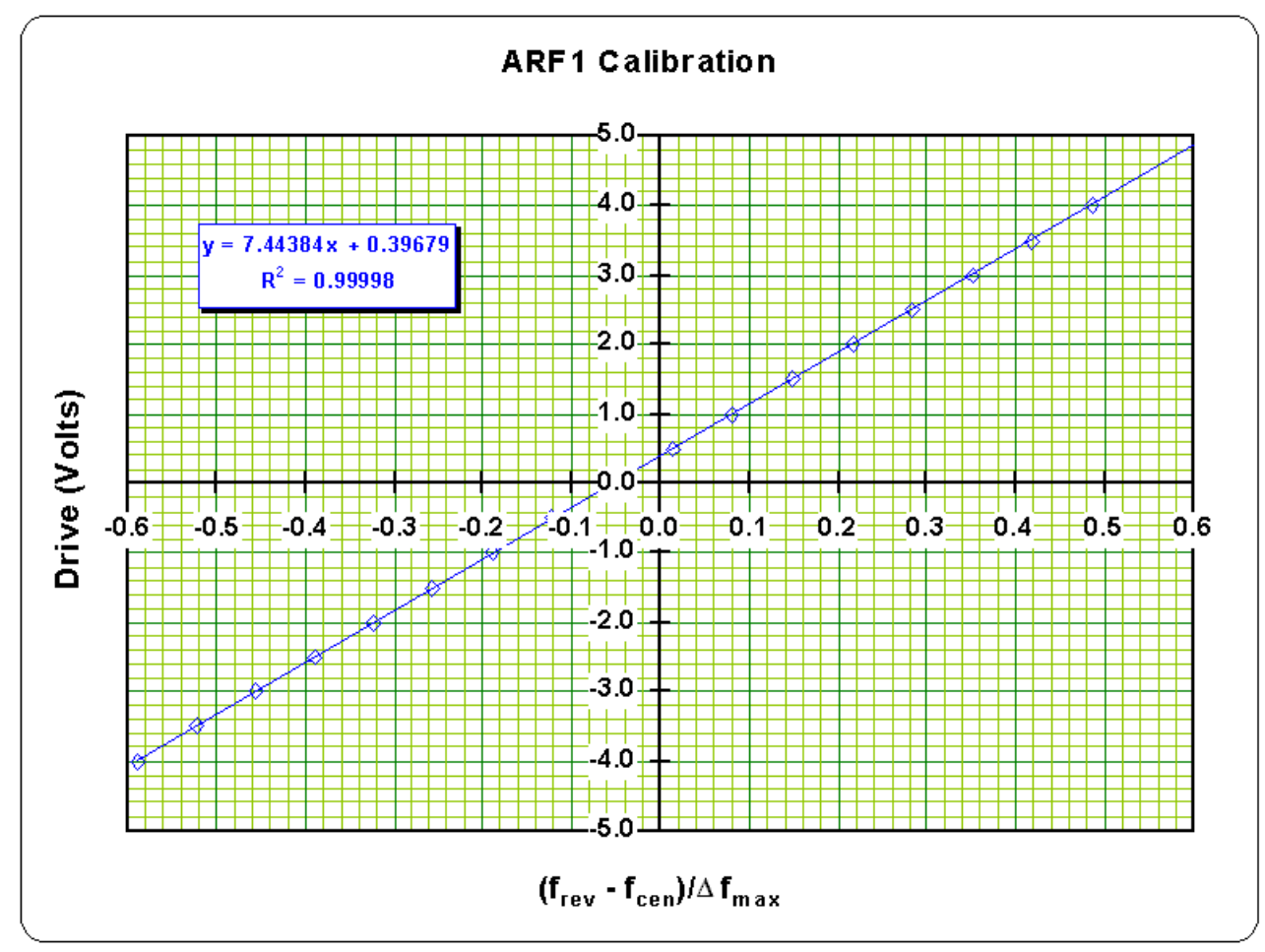

Figure $4 V_{d}$ versus $z$ from the April 18, 2001 ARF1 calibration measurements. $f_{\text {rev }}$ is the RF frequency measured on AP50 fanback divided by the harmonic number of ARF1 (84).

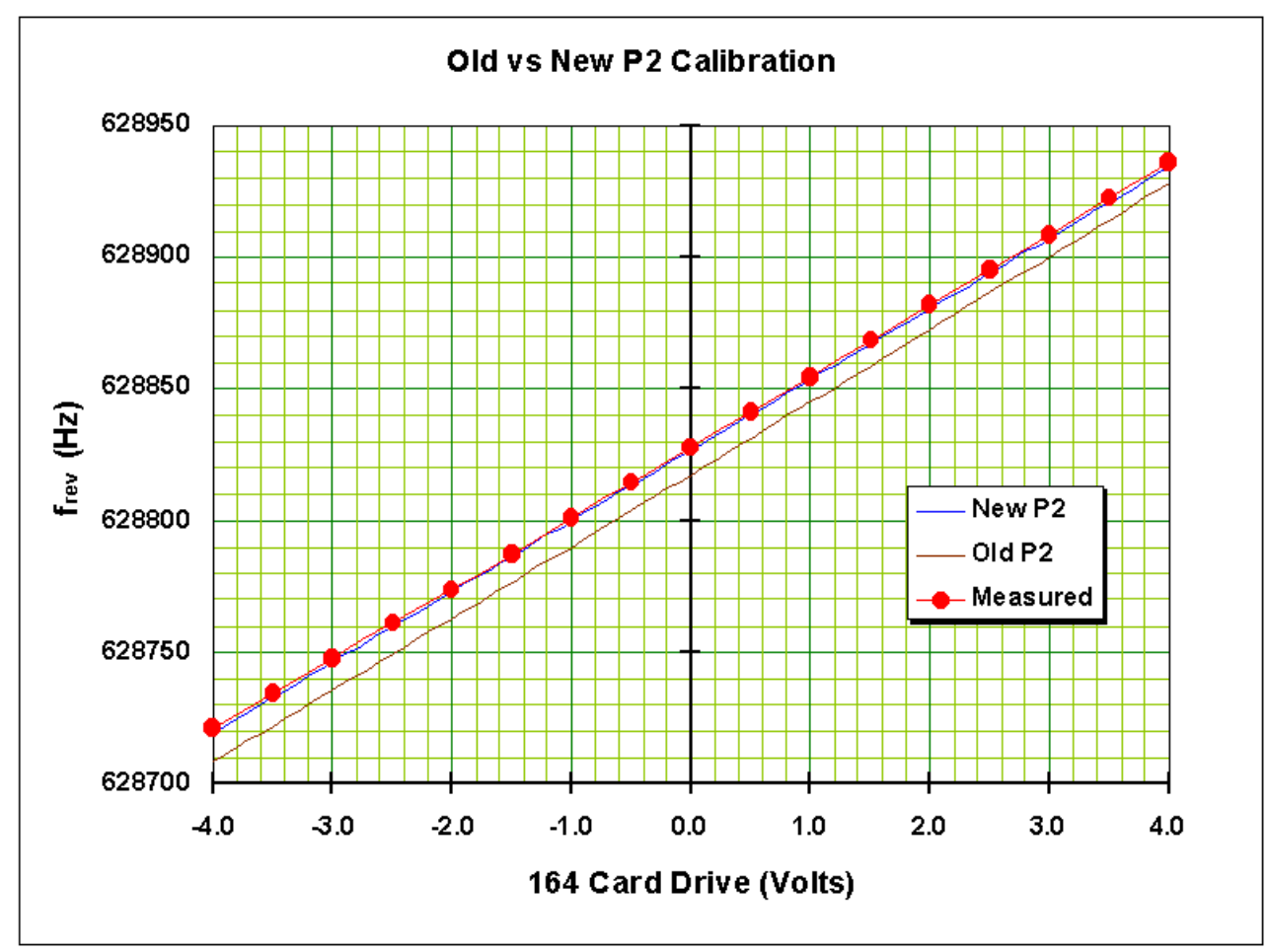

Figure 5 Comparison of the old P2 calibration (i.e. the constants from Figure 4 and the new constants. The Old and New P2 curves are obtained by solving equation 2) for $f_{\text {rev }}$. The $f_{\text {rev }}$ versus $V_{d}$ measurements of the April 18, 2001 calibration are also plotted. 


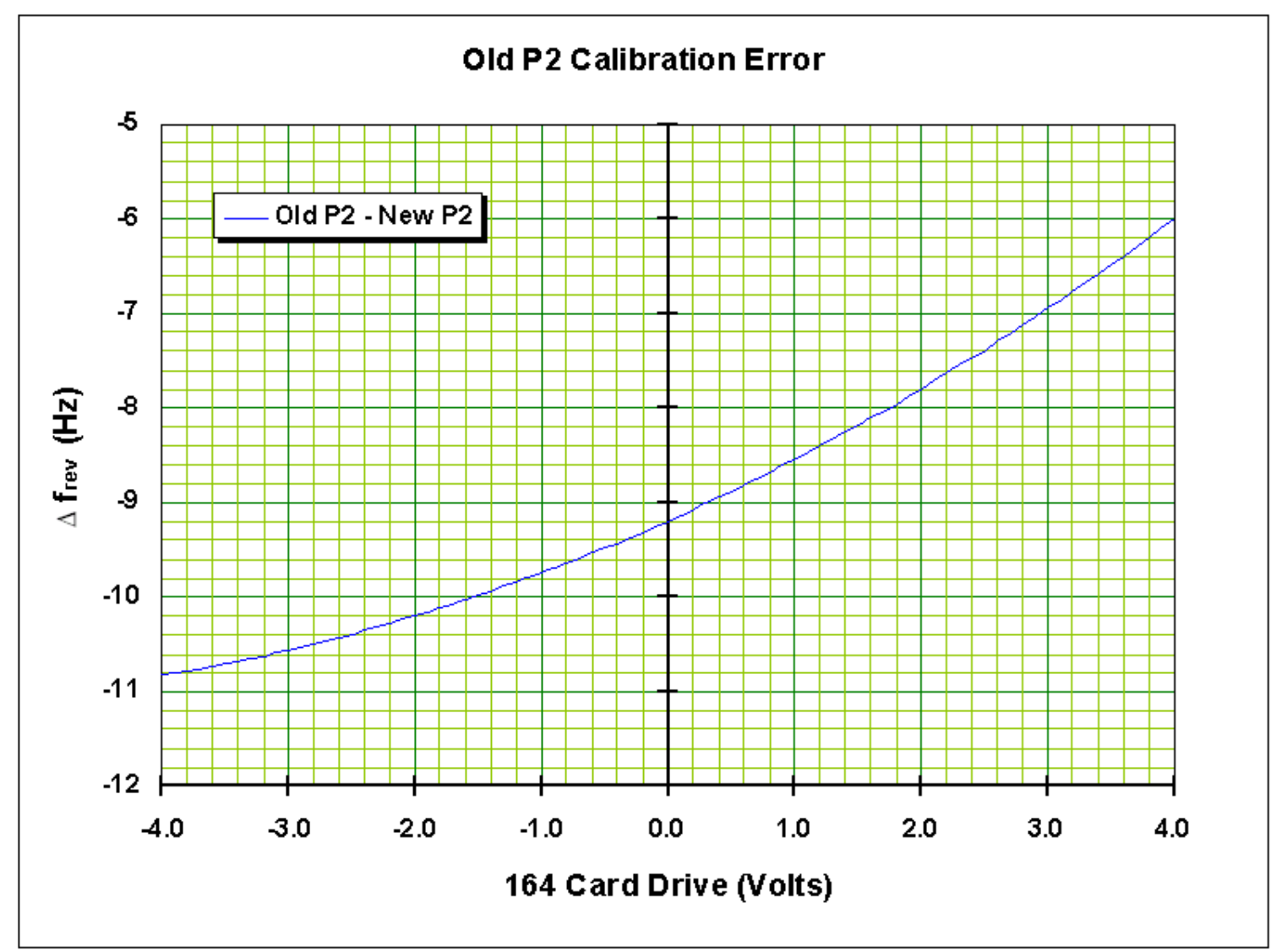

Figure 6 Frequency error (Predicted - Actual) as a function of low-level drive voltage. The injection orbit corresponds to a drive voltage of about -2.3 Volts. The central orbit corresponds to a drive voltage of about 0.5 Volts.

\section{Conclusions}

Here is a brief synopsis of the results of this report.

1. An analysis of the April 18, 2001 calibration measurements on ARF1 shows that the frequency and voltage constants in P2 are significantly in error.

2. Comparison of the actual voltage and frequency response to the low-level drive explain much of the "bad behavior" that has been observed from P2 generated ARF1 ramps.

3. New P2 calibration constants have been calculated from the ARF1 measurements, but have not yet been implemented. Since the ramps generated by P2 are generally "tweaked" to compensate for the deficiencies in the P2 calibration, inserting the new constants will require the modification of all of the ARF1 curves presently in use. 